\title{
ONE-STEP EXTRAPOLATION METHOD USING CHEBYSHEV RECURRENCE RELATION APPLIED TO SEISMIC MODELING
}

\author{
Laura Lara Ortiz ${ }^{1}$ and Reynam C. Pestana ${ }^{2}$
}

\begin{abstract}
In this work we show that the solution of the first-order differential wave equation for an analytical wavefield, using a finite-difference scheme in time, follows exactly the same recursion of modified Chebyshev polynomials. Based on this, we proposed a numerical algorithm for seismic modeling using the Chebyshev polynomial recursion applied on a pseudo-differential operator which can take care of any velocity variation. Exploiting the connection between the finite-difference scheme in time and the Chebyshev polynomial recursion, and considering the Fourier method to compute the spatial derivatives, we get the stability condition for this method taking into consideration only the range of validity of the Chebyshev polynomials. Thus, the proposed method can be used to march seismic wavefields in time generating a stable propagation of seismic waves free of numerical dispersion. To validate the obtained results with the one-step extrapolation method using Chebyshev polynomials, we compared the seismic responses of a two-layer model and a salt velocity model with the results of the rapid expansion method (REM) and also with the conventional finite-difference method. The results show that the quality of the seismic sections obtained using the proposed method is comparable to the results obtained with the aforementioned methods. Comparison of computational times shows that the one-step extrapolation method using Chebyshev polynomials takes a propagation time comparable with the recursive REM and finite-difference methods. The method proposed here was applied only for 2D seismic modeling but it can easily extend to 3D seismic modeling problems too.
\end{abstract}

Keywords: seismic modeling, acoustic wave equation, analytical wavefield, Chebyshev polynomials.

RESUMO. Neste trabalho, mostra-se que a solução da equação de onda de primeira ordem com um campo de onda analítico, usando um esquema de diferençasfinitas no tempo, segue exatamente a relação de recorrência dos polinômios modificados de Chebyshev. 0 algoritmo numérico proposto, ao ser aplicado à modelagem sísmica, utilizando a relação de recorrência dos polinômios de Chebyshev aplicada sobre um operador pseudodiferencial pode lidar com qualquer variação de velocidade. Explorando a conexão entre o esquema de diferenças-finitas no tempo, com a relação de recorrência dos polinômios de Chebyshev e o método de Fourier, para calcular as derivadas espaciais, foi possível encontrar a condição de estabilidade deste novo método levando em consideração apenas o intervalo de validade dos polinômios de Chebyshev. 0 algoritmo proposto pode ser utilizado para o avanço do campo de onda no tempo gerando uma propagação de ondas sísmicas estável e livre de dispersão numérica. Para validar os resultados obtidos com o método de extrapolação "One-step", usando a relação de recorrência dos polinômios de Chebyshev, comparamos as respostas sísmicas de um modelo de duas camadas e um modelo de velocidades que apresenta um corpo de sal com os resultados obtidos mediante 0 método de expansão rápida (REM) e o método das diferenças-finitas convencional. Os resultados mostram que a qualidade das seções sísmicas obtidas usando 0 método proposto é comparável com os resultados obtidos com os métodos de diferenças-finitas e o REM. A comparação dos tempos computacionais mostra que o método de extrapolação "One-step", com a relação de recorrência dos polinômios de Chebyshev, leva um tempo de propagação comparável com o tempo gasto pelo REM recursivo e o método de diferenças-finitas. Vale ainda ressaltar que o método proposto foi aplicado apenas para modelos sísmicos 2D, mas ele pode ser naturalmente estendido para a modelagem de dados sísmicos 3D.

Palavras-chave: modelagem sísmica, equação da onda acústica, campo analítico, polinômios de Chebyshev.

\footnotetext{
1 Universidade Federal da Bahia (UFBA), Centro de Pesquisa em Geofísica e Geologia (CPGG), Rua Barão de Jeremoabo, 123, Campus Universitário da Federação, 40170-115 Salvador, BA, Brazil. Phone: +55(71) 99161-7615 - E-mail: laura_lara_ortiz@hotmail.com

2Universidade Federal da Bahia (UFBA), Instituto de Física, Centro de Pesquisa em Geofísica e Geologia (CPGG), Departamento de Geofísica da Terra e do Meio Ambiente, Rua Barão de Jeremoabo, 123, Campus Universitário da Federação, 40170-115 Salvador, BA, Brazil. Phone: +55(71) 3283-8521; Fax: +55(71) 3283-8551 - E-mail: reynam@cpgg.ufba.br
} 


\section{INTRODUCTION}

Numerical modeling of wave equation has been widely used for the simulation of seismic data, inverting parameters of subsurface and reverse time migration. There has been a lot of efforts to produce stable numerical algorithms which are also free of dispersion noise. In practice, they are implemented by solving the acoustic wave equation using a finite-difference (FD) scheme. The explicit FD schemes are stable only when a limit on the size of the marching time is imposed. They also suffer from dispersion problems. Even in an isotropic medium, finite-difference approximations to the wave equation have a numerical phase and group velocities that are different from those of the true medium and vary with spatial frequency or wavenumber. As propagation proceeds in time and distance, the error in those velocities may accumulate to an unacceptable amount that results in the distortion of the resonances in the frequency domain. Traditionally, this error is called numerical dispersion and can be suppressed by using either higher-order temporal and spatial schemes or by increasing temporal or spatial resolution. Any of the cases leads to an increase in computational cost (Zhang \& Zhang, 2009) and therefore, methods that remain stable and free of dispersion noise are needed.

Pseudospectral methods, where the spatial derivatives are evaluated in Fourier domain, can help reduce these numerical problems because they provide optimal accuracy for the given grid size. Pseudospectral methods implemented in the Fourier domain provide computational efficiency and a high degree of accuracy (Kosloff \& Baysal, 1982; Reshef et al., 1988).

Another very efficient solution, initially presented by Kosloff et al. (1989), is the rapid expansion method (REM), which has been applied with success in seismic modeling and reverse time migration. This method is essentially a variation of the method proposed by Tal-Ezer (1986). In REM, modified Chebyshev polynomials are incorporated to expand the cosine operator that appears on the wave equation solution, and is the base for a time stepping scheme for both small and large time step (Pestana \& Stoffa, 2010). Another highlight of this method is that, it is stable and free of dispersion numerical noise when implemented together with the Fourier method.

A recent method introduced in the geophysical literature to solve the acoustic wave equation is the one-extrapolation method (OSE) (Zhang \& Zhang, 2009). In the OSE a complex wavefield is introduced and a square-root operator is defined. By this way, the two-way wave equation can be formulated as a first-order partial differential equation in time, which is similar to the one-way wave equation in depth. To solve the new first-order differential wave equation, (Zhang \& Zhang, 2009) used a stable explicit extrapolation method in time based on an optimized separable approximation (OSA) proposed by Song (2001).

In the REM approach, Pestana \& Stoffa (2010) show that the result of expanding the cosine function using Chebyshev polynomials has the same form of a Taylor-series expansion when a specific analytical expression for the Bessel function is considered. Besides that, they also showed that if only two terms in the Chebyshev expansion is used, the expression is reduced to a second-order finite-difference time approximation.

Starting from this relation between REM and FD method, we show in this paper that the FD approximation in time of the firstorder differential equation follows the recursion of the Chebyshev polynomials when applied on a square-root pseudo-differential operator. Using the pseudospectral method for spatial derivatives and the connection between Chebyshev recurrence relation and finite-difference approximation in time, we find the stability condition exploring only the range of validity of the Chebyshev polynomials. The stability condition is exactly the same one obtained for the conventional pseudospectral method recommended by Baysal et al. (1983).

Thus, we obtain a finite-difference scheme for the one-step extrapolation method using the Chebyshev polynomial recurrence and the proposed numerical algorithm can be used to march the complex wavefield in time generating the stable propagation of seismic waves free of numerical dispersion.

To demonstrate the relation between the FD approximation in time and the modified Chebyshev Polynomial recursion, two models are used and the impulse response and time-sections are compared with the conventional REM and with the conventional FD for the full-wave equation. The results obtained are equivalent to the results obtained with these cited methods, stable and free of dispersion for a time step limited by the stability condition. The method here proposed was only tested on 2D models but it can be naturally extended and applied to 3D seismic modeling problems.

\section{THEORY}

\section{Acoustic wave equation}

The acoustic wave equation for a $2 \mathrm{D}$ media is expressed in terms of a second-order in time differential partial equation:

$$
\frac{1}{v^{2}(\mathbf{x})} \frac{\partial^{2} p(\mathbf{x}, t)}{\partial t^{2}}-\nabla^{2} p(\mathbf{x}, t)=0
$$

where $(\mathbf{x})=(x, z)$ is the position vector, $p(\mathbf{x}, t)$ is the pressure field, $v(\mathbf{x})$ is the propagation velocity of the acoustic wave in the medium, and $\nabla^{2}=\frac{\partial^{2}}{\partial x^{2}}+\frac{\partial^{2}}{\partial z^{2}}$ is the Laplacian operator 
in Cartesian coordinates.

Defining an operator $-L^{2}$, such that

$$
-L^{2}=v^{2}(\mathbf{x})\left(\frac{\partial^{2}}{\partial x^{2}}+\frac{\partial^{2}}{\partial z^{2}}\right) .
$$

and introducing a source term $S(\mathbf{x}, t)$, equation 1 can be rewritten as

$$
\frac{\partial^{2} p(\mathbf{x}, t)}{\partial t^{2}}+L^{2} p(\mathbf{x}, t)=S(\mathbf{x}, t) .
$$

\section{One-Step extrapolation method}

The one-step extrapolation method of Zhang \& Zhang (2009) presents an alternative way to obtain the solution of the wave equation. First, the full-wave equation is transformed into a first-order partial differential equation, similar to the one-way wave equation. In this formulation proposed by Zhang \& Zhang (2009), the real pressure wavefield is replaced by a complex pressure field. Similar wave equation was also derived by Gazdag (1981), but with a real wavefield, and it was first used by Baysal et al. (1983) for time extrapolation and solved by the pseudospectral method. This new first-order differential wave equation is equivalent to the two-way wave equation (Eq. 1) and produces the same traveltime and leading order amplitude as the conventional wave equation (Zhang \& Zhang, 2009).

For a medium with $2 \mathrm{D}$ constant velocity, $v(\mathbf{x})=c$, Eq. (1) is transformed to the wave number domain, through a spatial Fourier transform, resulting:

$$
\left(\frac{\partial^{2}}{\partial t^{2}}+\varphi^{2}\right) \hat{p}(\mathbf{k}, t)=0
$$

where $\varphi=v \sqrt{k_{x}^{2}+k_{z}^{2}}$ and $\mathbf{k}=\left(k_{x}, k_{z}\right)$ is the spatial wave number vector.

Instead of using a real wavefield $p(\mathbf{x}, t)$, Zhang \& Zhang (2009) uses a complex wavefield $P(\mathbf{x}, t)$, such that:

$$
P(\mathbf{x}, t)=p(\mathbf{x}, t)+i q(\mathbf{x}, t)
$$

where $q(\mathbf{x}, t)$ is the Hilbert transform of $p(\mathbf{x}, t)$, which in the frequency domain defined as:

$$
\hat{q}(\mathbf{x}, \omega)=i \operatorname{sgn}(\omega) \hat{p}(\mathbf{x}, \omega)=\frac{i \omega}{|\omega|} \hat{p}(\mathbf{x}, \omega)
$$

Now, using the dispersion relation $\omega^{2} / v^{2}=k_{x}^{2}+k_{z}^{2}$, from wave equation (Eq. 1), the Hilbert wavefield (Eq. 6) can be expressed as:

$$
\hat{\hat{q}}(\mathbf{k}, \omega)=\frac{i \omega}{v \sqrt{k_{x}^{2}+k_{z}^{2}}} \hat{\hat{p}}(\mathbf{k}, \omega)=\frac{i \omega}{\varphi} \hat{\hat{p}}(\mathbf{k}, \omega)
$$

and transforming back to the time domain, we obtain:

$$
\hat{q}(\mathbf{k}, t)=\frac{1}{\varphi} \frac{\partial \hat{p}(\mathbf{k}, t)}{\partial t}
$$

Thus, Eq. (4) is equivalent to the following system of equations (Zhang \& Zhang, 2009)

$$
\frac{\partial}{\partial t}\left(\begin{array}{c}
\hat{p} \\
i \hat{q}
\end{array}\right)=\left(\begin{array}{cc}
0 & -i \varphi \\
-i \varphi & 0
\end{array}\right)\left(\begin{array}{l}
\hat{p} \\
i \hat{q}
\end{array}\right)
$$

or, in a simpler way:

$$
\frac{\partial \hat{P}}{\partial t}=\frac{\partial(\hat{p}+i \hat{q})}{\partial t}=-i \varphi(\hat{p}+i \hat{q})=-i \varphi \hat{P}
$$

The complex pressure field $P(\mathbf{x}, t)$ satisfies the following firstorder partial differential equation in time domain, i.e.

$$
\left(\frac{\partial}{\partial t}+i \Phi\right) P(\mathbf{x}, t)=0
$$

where $\Phi$ is a pseudo-differential operator, defined in the space domain as:

$$
\Phi=v(x, z) \sqrt{-\nabla^{2}}
$$

or symbolically given by:

$$
\varphi=v(x, z) \sqrt{k_{x}^{2}+k_{z}^{2}}
$$

If the velocity is constant, the solution of Eq. (11) can be expressed as:

$$
P(\mathbf{x}, t+\Delta t)=\left(F^{-1} e^{-i v \sqrt{k_{x}^{2}+k_{z}^{2}} \Delta t} F\right) P(\mathbf{x}, t)
$$

where $F$ and $F^{-1}$ represent the direct and inverse Fourier transforms, respectively.

For a variable velocity, the solution of Eq. (11) can be written symbolically as:

$$
P(\mathbf{x}, t+\Delta t)=e^{-i \Phi \Delta t} P(\mathbf{x}, t)
$$

Recall that the solution of the one-way wave equation proposed by Gazdag (1978), Claerbout (1971), has the following form:

$$
D(\mathbf{z}+\Delta z)=e^{-i \Lambda \Delta z} D(z)
$$

where $\Lambda$ is the square-root operator defined:

$$
\Lambda=\sqrt{\frac{\omega^{2}}{v^{2}(x, z)}-k_{x}^{2}}
$$

Comparing Equations (15) and (16) are similar and therefore different extrapolation methods used to calculate the one-way operator $e^{i \Lambda \Delta z}$ can be applied in the solution of Eq. (15), taking into account that in this case, the propagation is performed in time. 
For OSE method, Zhang \& Zhang (2009) implemented it numerically using a solution based on an optimized separable approximation (OSA), proposed by Song (2001), which approximates the operator $e^{-i \varphi \Delta t}$ as follows:

$$
e^{-i \varphi \Delta t}=\sum_{n=1}^{N} a_{n}(V) b_{n}(k),
$$

where $a_{n}(V)$ and $b_{n}(k)$ are complex functions that depend on the wave speed and wave number, respectively. These functions can be determined by computing the left and right eigenvalues of the two dimension function

$$
e^{-i V k \Delta t}, \quad \text { where } \quad V \in\left[v_{\min }, v_{\max }\right]
$$

and

$$
k=\sqrt{k_{x}^{2}+k_{z}^{2}}, \in\left[k_{\min }, k_{\max }\right] .
$$

After obtaining $a_{n}(V)$ and $b_{n}(k)$ by the OSA method, the algorithm proposed by Zhang \& Zhang (2009) requires, for every time step extrapolation, one Fast Fourier Transform (FFT) and $N$ fast inverse transforms. However, neither the number of FFTs nor the stability criterion about the OSA method are explicitly determined.

\section{One-step extrapolation method using the Chebyshev polynomials}

An alternative way to solve the full-wave equation, based on the one-step extrapolation method (Zhang \& Zhang, 2009), is given here, where we use the recurrence relation of the modified Chebyshev polynomials to approximate the extrapolation operator in time in a stable solution.

Using the complex field $P(x, z, t)=p(x, z, t)+$ $i H[p(x, z, t)]$, as proposed by Zhang \& Zhang (2009), where $H[$.$] is the Hilbert transform operator, we have that the complex$ pressure field $P(\mathbf{x}, t)$ satisfies the following first-order partial differential equation in time

$$
\frac{\partial P(x, z, t)}{\partial t}=\Phi P(x, z, t)
$$

where $\Phi$ is a pseudo-differential operator, but now defined as:

$$
\phi=-i v(x, y) \sqrt{k_{x}^{2}+k_{z}^{2}}
$$

Then, according to the finite-difference method for the derivative of the wavefield $P$ in time, we have:

$$
\begin{gathered}
P(x, z, t+\Delta t)= \\
2 \Delta t \frac{\partial P(x, z, t)}{\partial t}+P(x, z, t-\Delta t) .
\end{gathered}
$$

Then, substituting Eq. (19) into Eq. (21) and writing in a simplified form, we find that:

$$
P_{n+1}=2 \Delta t \Phi P_{n}+P_{n-1} \quad \text { for } n=1,2,3 \ldots
$$

Here, we have that $P(n \Delta t)=P_{n}$, where $\Delta t$ is the time step.

Thus, from Eqs. (19) and (13), the time derivative of $P$ for each time step is numerically computed by applying the Fourier method and then it is approximated using a centered finitedifference scheme (Eq. 21).

\section{Chebyshev polynomials}

Let's first introduce the Chebyshev polynomials which are used to solve a wide range of problems in many different areas of physics and engineering.

The Chebyshev polynomials are defined as:

$$
T_{n}(x)=\cos (n \theta), \quad \text { where } \quad x=\cos (\theta)
$$

and they satisfy the following recurrence relation

$$
T_{n+1}(x)=2 x T_{n}(x)-T_{n-1}(x)
$$

which is very useful in computing the polynomials from $T_{0}(x)=1$ and $T_{1}(x)=x$.

When the Chebyshev argument is an imaginary number $z=i x$, we have the modified Chebyshev polynomials and they satisfy the following recurrence

$$
Q_{n+1}(z)=2 z Q_{n}(z)+Q_{n-1}(z) .
$$

The modified Chebyshev polynomials are defined as $Q_{n}(z)=$ $i^{n} T_{n}(x)$, where $T_{n}(x)$ are the ordinary Chebyshev polynomials. Again, the recursion is initiated by: $Q_{0}(z)=1$ and $Q_{1}(z)=z$.

Now, we show that the solution of the first-order wave equation (Eq. 19), using a finite-difference scheme in time, given by Eq. (22), has the same similarity with the Chebyshev polynomials found for the full-wave equation in the rapid expansion method (REM) as showed by Pestana \& Stoffa (2010).

Recalling the recurrence relation for modified Chebyshev polynomials, $Q_{n+1}(z)=2 z Q_{n}(z)+Q_{n-1}(z)$ and comparing it with the finite-difference solution in time (Eq. 22), we note that the finite-difference wave fields are just the Chebyshev polynomials in $\Phi \Delta t$ acting on the initial wavefield $P_{o}$.

Thus, we can propose the following recursive solution to the first-order differential wave equation (Eq. 19) as:

$$
P_{n+1}=Q_{n+1}(\Phi \Delta t) P_{0} \quad \text { for } n=1,2,3 \ldots
$$


with the first terms defined as:

$$
\begin{gathered}
Q_{0}(\Phi \Delta t) P_{0}=P_{0} \\
Q_{1}(\Phi \Delta t) P_{0}=(\Phi \Delta t) P_{0}
\end{gathered}
$$

These two first terms are calculated following the Chebyshev polynomial recurrence (expression 25), where they are defined as $Q_{0}(z)=1$ and $Q_{1}(z)=z$, with $z=\Phi \Delta t$. In general, all wavefields can be generated using Eq. (26), where the polynomials satisfy the recurrence relation given by expression 25 .

The stability of this new method is related to the validity interval of the Chebyshev polynomials. Since $Q_{n}(z)$ is limited to the interval $[-i, i]$ for all $n$, so we have that this scheme is stable if and only if:

$$
\Delta t<\frac{1}{R}
$$

where $R$ is the maximum value associated with $\Phi$, the pseudospectral operator, which for the 2-D case is given by:

$$
R=\pi v_{\max } \sqrt{\left(\frac{1}{\Delta x}\right)^{2}+\left(\frac{1}{\Delta z}\right)^{2}}
$$

Therefore, one-step extrapolation method (OSE) using the modified Chebyshev polynomials is stable, if the time step $\Delta t$ satisfies the above criteria, i.e., $\Delta t R<1$. Thus, exploring the relationship between the finite-difference method and the recurrence relation of Chebyshev polynomials, we can see that on a mesh where $\Delta x=\Delta z$, the limit of stability is given by:

$$
\alpha=\frac{v_{\max } \Delta t}{\Delta x}<0.2,
$$

which is exactly the stability condition for the pseudospectral method as recommended by Baysal et al. (1983).

The method present here was derived and tested only for 2D case as we show in the next section, but it can be easily extend for 3D case and applied for modeling of 3D complex structural models.

\section{MODELING EXAMPLES}

\section{Two-layer synthetic velocity model}

The first synthetic velocity model used is formed by two flat layers. The velocity in the upper and lower layer is $2000 \mathrm{~m} / \mathrm{s}$ and $3500 \mathrm{~m} / \mathrm{s}$ respectively. The numerical grid consists of $260 \times 260$ nodes in total. The spatial sampling in the velocity grid is $10 \mathrm{~m}$ in both directions. The source time signal is a $40 \mathrm{~Hz}$ Ricker wavelet applied at the middle of the model in the position $x_{s}=1300 \mathrm{~m}$ and $z_{x}=1300 \mathrm{~m}$. The time step size used was $1 \mathrm{~ms}$ with $0.6 \mathrm{~s}$ total propagation time, satisfying the dispersion and stability criteria. The absorbing boundaries of 50-grid points width at the borders of the numerical grid (Cerjan et al., 1985) were applied.

To show the advantages and disadvantages related with the efficiency and accuracy of the method presented in this work, we take an instantaneous of the wavefield (snapshot) at $t=$ $0.30 \mathrm{~s}$. Figure 1 (a) shows the snapshot for the one-step extrapolation method using Chebyshev polynomials. For comparison, we present snapshots, at the same instant of time, but computed by different methods: Figure 1(b) recursive REM (Pestana \& Stoffa, 2010), in Figure 1(c) using the non-recursive REM (Tessmer, 2011) and in Figure 1(d) by the conventional finite-difference.

From these results we can see that there is a great similarity between them, and all methods display the same seismic events despite being obtained by different numerical approaches and algorithms. We can also note that the results are stable and free of numerical dispersion. From Figure 1(a) we can clearly see that the wave fronts are better defined, showing that modeling using the one-step extrapolation method with Chebyshev polynomials provide higher resolution.

\section{Salt synthetic velocity model}

In order to verify the accuracy and efficiency of these methods for modeling complex structures, we tested all methods using a salt model velocity as shown in Figure 2. This model is defined on a mesh of $338 \times 210$ with a grid spacing of $10 \mathrm{~m}$ along the transverse and depth direction. The point source used was a Ricker wavelet with maximum frequency of $30 \mathrm{~Hz}$, which was injected at the coordinates $1689 \mathrm{~m}$ in horizontal and $1100 \mathrm{~m}$ in vertical. The source was located below the salt body just to have a better view of wave propagation passing through the salt body. We used a time step size of $2 \mathrm{~ms}$ for a total propagation time of $1.2 \mathrm{~s}$.

Each image shown from Figure 3(a) to Figure 3(d) represents a wavefield snapshot at $t=0.26 \mathrm{~s}$ for the four methods used at the last section in the same order, respectively. On these snapshots we can observe the propagation of the wave front and its effects due to the high velocity contrast passing across the body salt. The wavefield travels with greater velocity within the body salt and interacts with the complex structures inside and outside thereof. A few reflections inside the salt body is clearly observed when the wave reaches the edge of the upper body salt.

To make a better comparison of the results, the same spatial parameters and the total recording time used to implement the different modeling methods based on REM were used in the onestep extrapolation method using Chebyshev polynomials. With respect to the temporal sampling interval in order to guarantee 


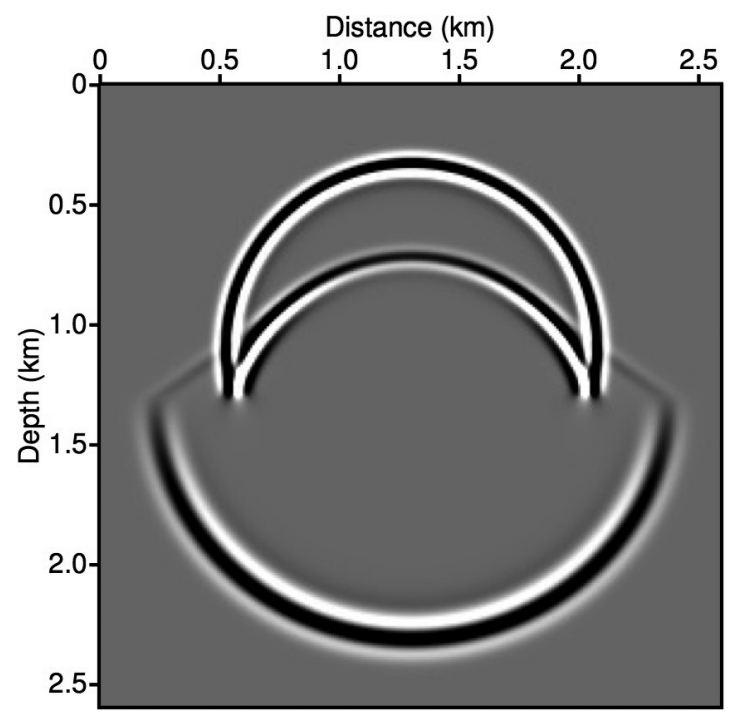

(a)

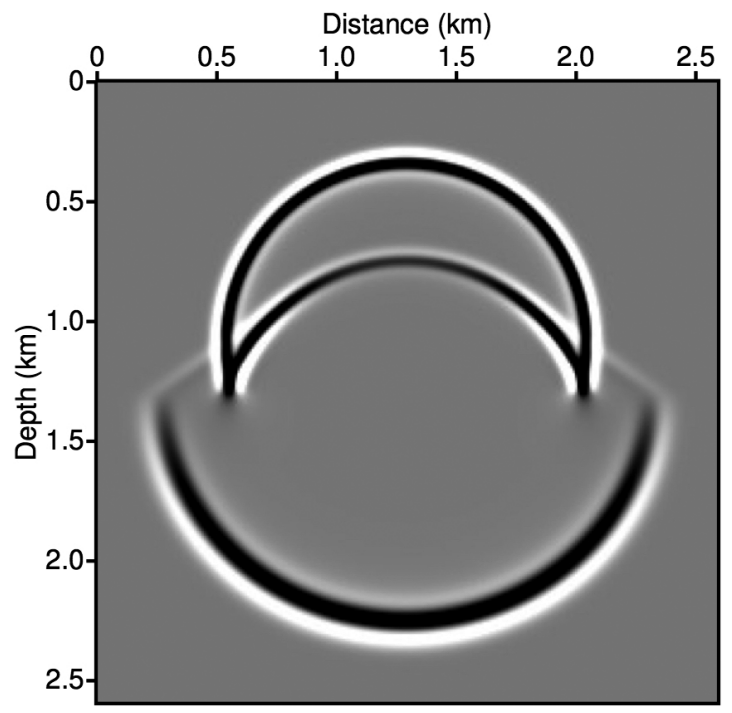

(c)

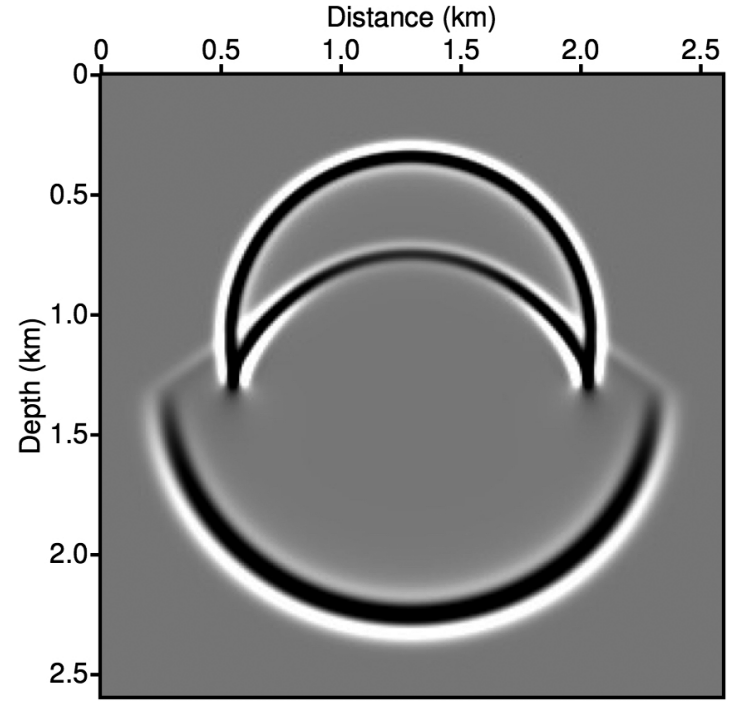

(b)

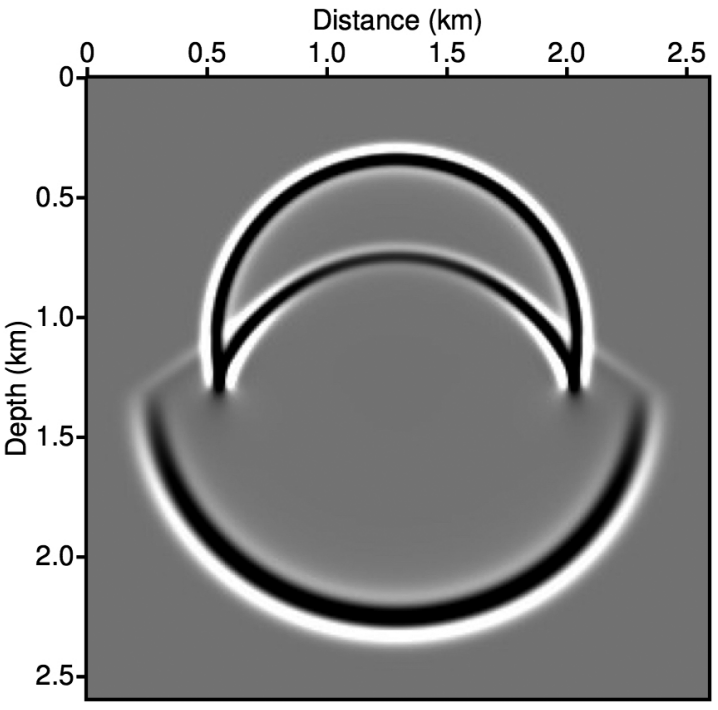

(d)

Figure 1 - Wavefield snapshots at $0.26 \mathrm{~s}$ for a two-layer velocity model computed by different methods. (a) One-step extrapolation method using Chebyshev polynomials; (b) Recursive REM; (c) Non-recursive REM; (d) Finite-difference method.

the method stability, in the OSE with Chebyshev polynomials, the time step for time extrapolation is calculated based on the input parameters, that means, the method seeks to ensure that the argument of the Chebyshev polynomials in the range of $[-1,1]$, respecting the stability condition $\Delta t<1 / R$, where the value of $R$ is defined in Eq. (29). In this case, the value of $\Delta t$ is automatically calculated by taking into account the initial parameters and results in a value of $0.560 \mathrm{~ms}$. It is also observed that the results are equivalents: they are stable and free of dispersion noise, showing the direct connection between the one-step extrapolation method with the REM using the modified Chebyshev polynomials.

In order to obtain the respective seismogram, the source was positioned at $x=1690 \mathrm{~m}$ and $z=10 \mathrm{~m}$. To generate the time section, receivers were located in the same horizontal position as the source. The seismogram from the seismic modeling using the one-step extrapolation with Chebyshev polynomials for the salt velocity model is shown in Figure 4(a). Comparing the results 

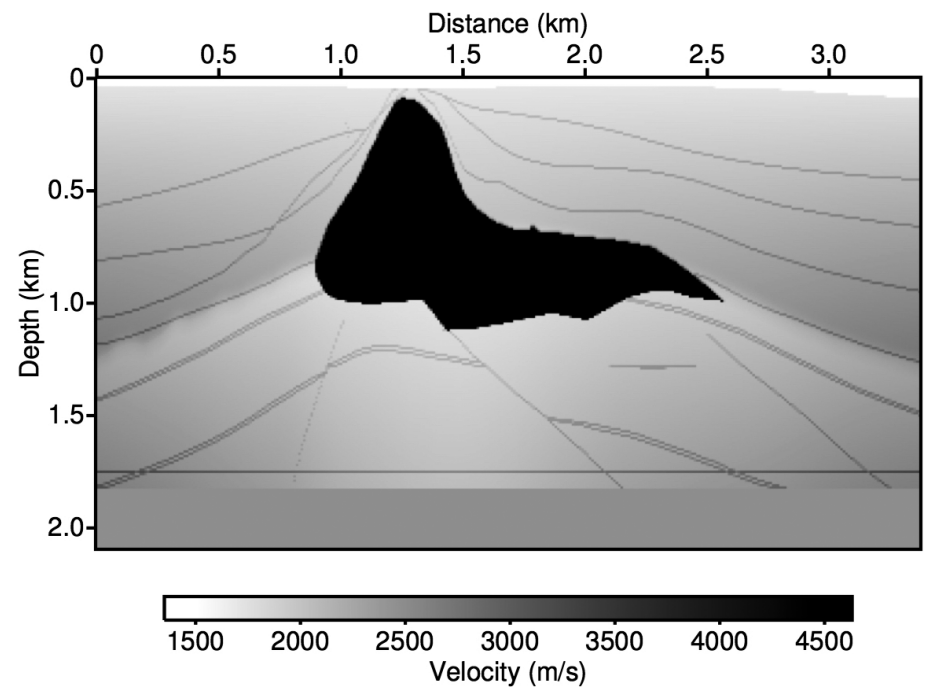

Figure 2 - Salt velocity model.

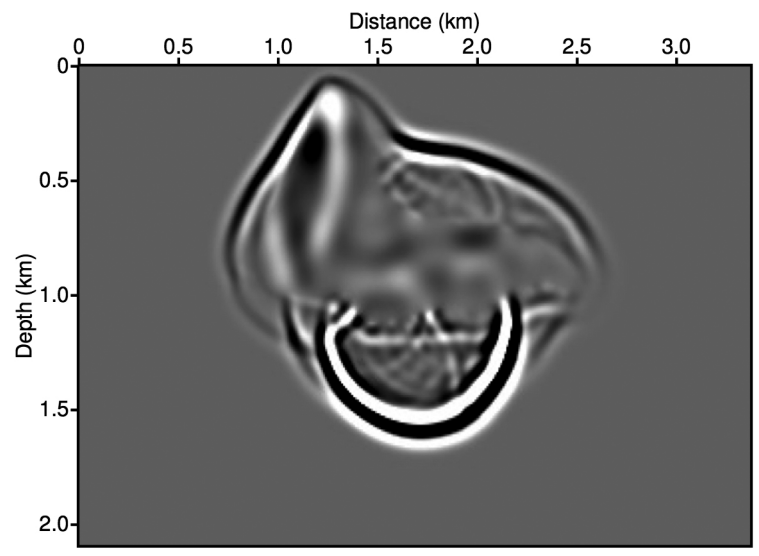

(a)

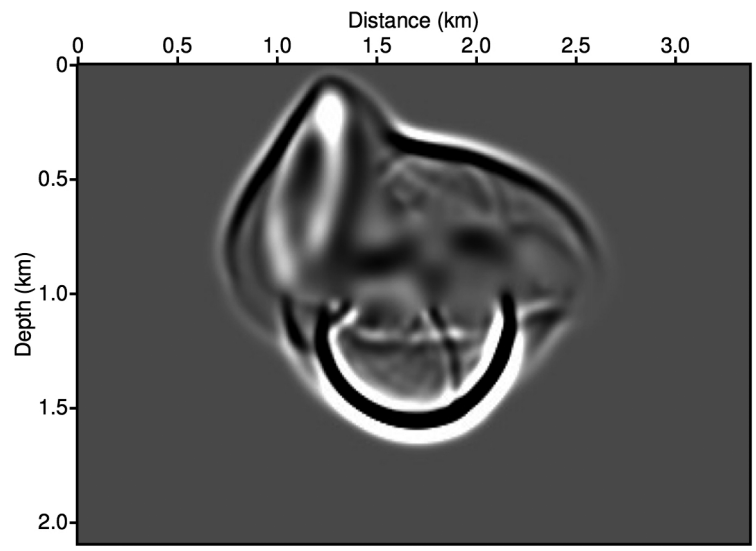

(c)

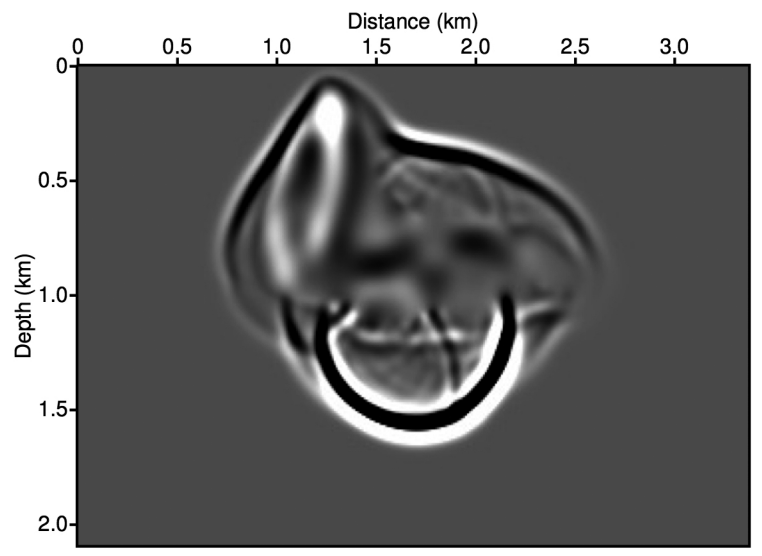

(b)

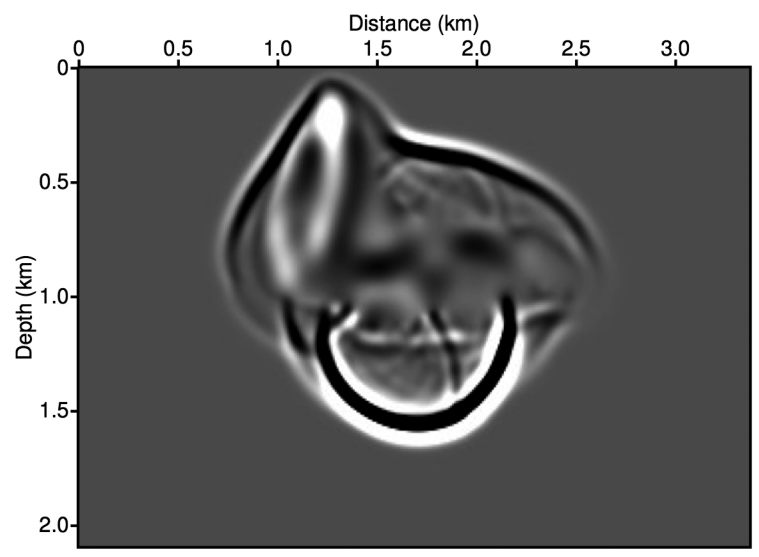

(d)

Figure 3 - Wavefield snapshots at $0.30 \mathrm{~s}$ for a salt model computed by different methods. (a) One-step extrapolation method using Chebyshev polynomials; (b) Recursive REM; (c) Non-recursive REM; (d) Finite-difference method. 


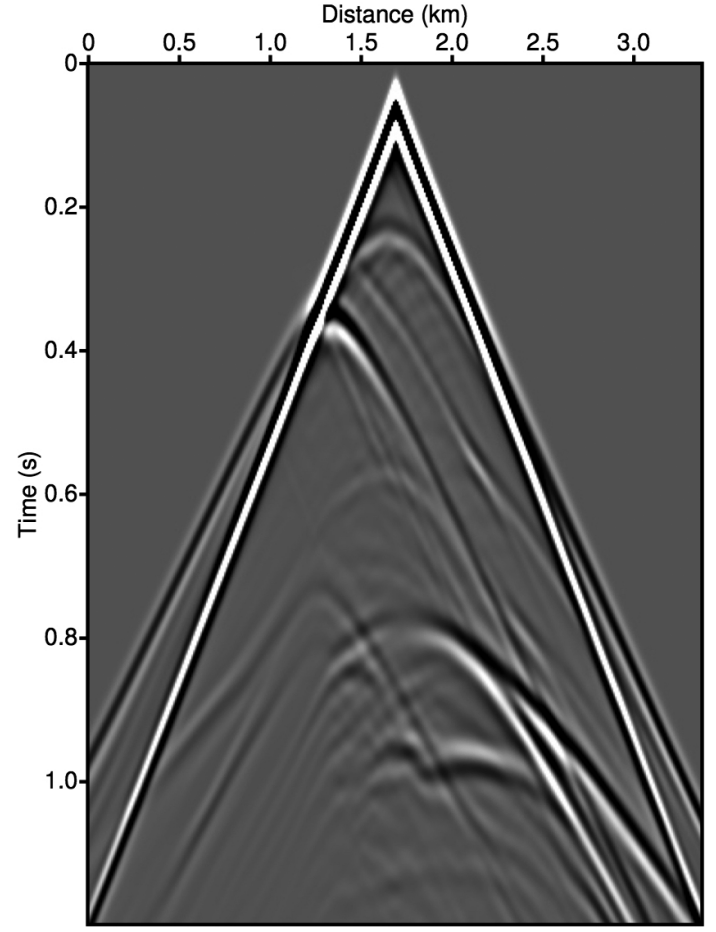

(a)

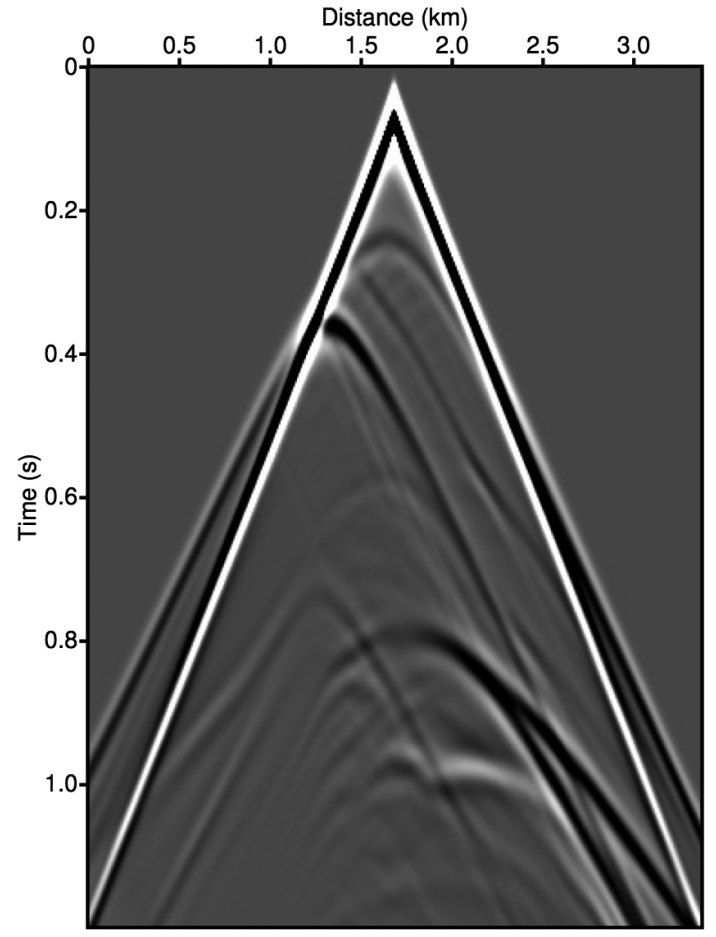

(c)

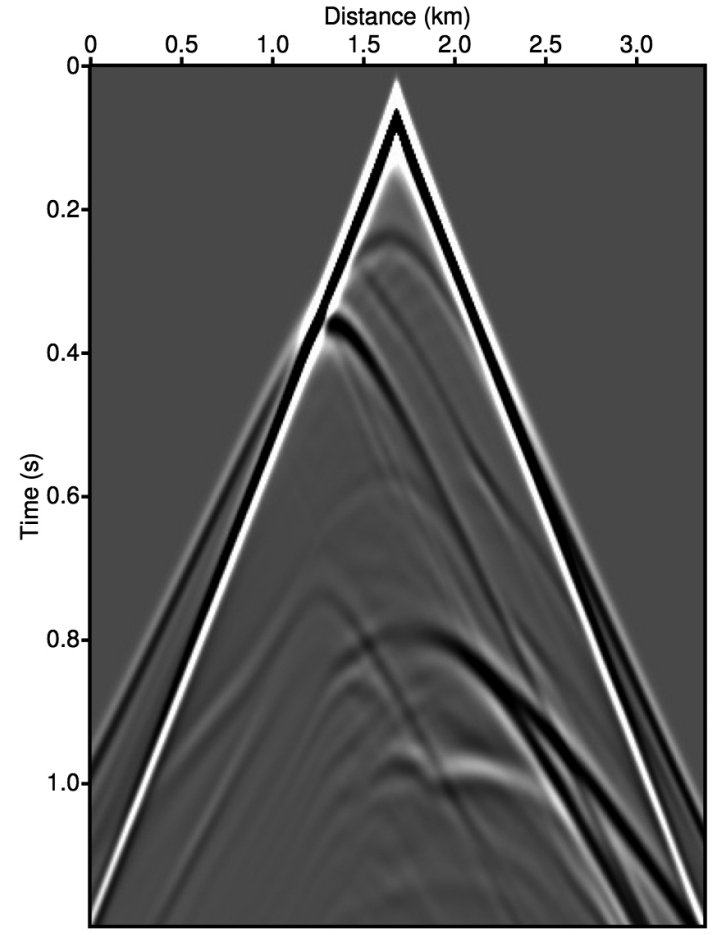

(b)

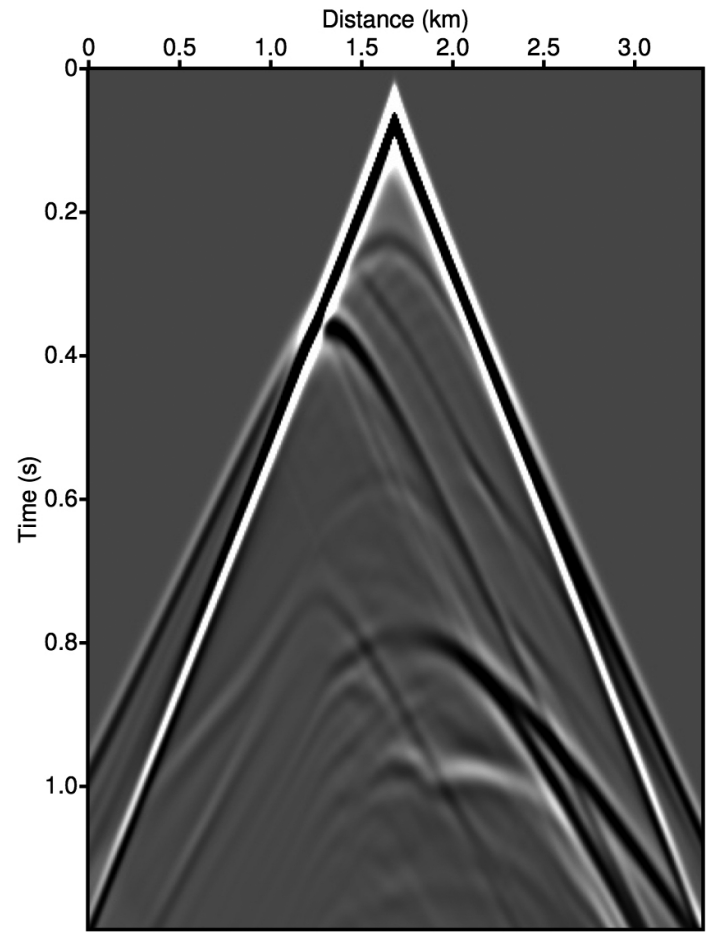

(d)

Figure 4 - Synthetic seismogram for a salt model computed by different methods. (a) One-step extrapolation method using Chebyshev polynomials; (b) Recursive REM; (c) Non-recursive REM; (d) Finite-difference method. 
shown in Figures 4(b), 4(c) and 4(d), which represent the seismogram of recursive REM, non-recursive REM and finite-difference method, respectively, we can notice that they are all equivalents. Besides that, similarity between them in the presentation of seismic events generated during the propagation wave field is demonstrated and the OSE method using the Chebyshev polynomials is validated by this comparison.

By this way, it can be said that all modeling methods implemented propagate the pressure wave field in a precise way and the methods are stable and free from numerical dispersion, demonstrating in this way the applicability and efficiency of the proposed algorithm to seismic modeling purpose in complex media.

From theses synthetics results, we also noticed that the One-step extrapolation method using the recurrence relation of the Chebyshev polynomials produced images with a much better resolution when compared with the other modeling results.

As can be seen, the differences between the quality of images obtained by the different methods presented is practically non-existent, therefore, we take into account the running time between the different modeling methods as another parameter in the analysis. The computational cost under the same hardware and software conditions are notable different. For instance, the results showed that the one-step extrapolation using Chebyshev polynomials method took a similar processing time to the recursive REM and finite-difference method, but, the non-recursive REM is the method that has spent most execution time. The numerical tests also showed that non-recursive REM took almost six times more that the recursive REM.

\section{CONCLUSION}

We present a new method that uses the recurrence relation of Chebyshev polynomials to solve the final equation presented by the One-step extrapolation method. The quality of images obtained by this new method was compared with the results obtained with the recursive and non-recursive REM and the finite-difference method. These results showed that the proposed method is stable and free of numerical dispersion, and can display the same seismic events presented in the others methods. By this way, we demonstrated the efficiency and applicability of this method for modeling seismic data from complex structures, with strong lateral perturbations of slowness, like the salt body. In computational terms, the computational time required for modeling is also comparable with the others methods. The presented method was efficient when compared with the modeling time using the recursive REM and the finite-difference method.

\section{ACKNOWLEDGMENTS}

This research was supported by CNPq and INCT-GP/CNPq. The authors also acknowledge the facility support from CPGG/UFBA and the comments and suggestions from the anonymous reviewers.

\section{REFERENCES}

BAYSAL E, KOSLOFFDD \& SHERWOOD JW. 1983. Reverse time migration. Geophysics, 48: 1514-1524.

CERJAN C, KOSLOFF D, KOSLOFF R \& RESHEF M. 1985. A nonreflecting boundary condition for discrete acoustic and elastic wave equations. Geophysics, 50: 705-708.

CLAERBOUT JF. 1971. Toward a unified theory of reflector mapping. Geophysics, 36: 467-481.

GAZDAG J. 1978. Wave equation migration with the phase-shift method. Geophysics, 43: 1342-1351.

GAZDAG J. 1981. Modeling of the acoustic wave equation with transform methods. Geophysics, 46: 854-859.

KOSLOFF DD \& BAYSAL E. 1982. Forward modeling by a Fourier method. Geophysics, 47: 1402-1412.

KOSLOFFD, QUEIROZ FILHO A, TESSMERE \& BEHLE A. 1989. Numerical solution of the acoustic and elastic wave equations by a new rapid expansion method. Geophysical Prospecting, 37: 383-384.

PESTANA R \& STOFFA P. 2010. Time evolution of the wave equation using rapid expansion method. Geophysics, 75: T121-T131.

RESHEF M, KOSLOFF D, EDWARDS M \& HSIUNG C. 1988. Threedimensional acoustic modeling by the Fourier method. Geophysics, 53: 1175-1183.

SONG J. 2001. The optimized expression of a high dimensional function/manifold in a lower dimensional space. Chinese Scientific Bulletin, 46: 977-984.

TAL-EZER H. 1986. Spectral methods in time for hyperbolic problems. SIAM Journal on Numerical Analysis, 23: 11-26.

TESSMER E. 2011. Using the rapid expansion method for accurate time-stepping in modeling and reverse-time migration. Geophysics, 76 : S177-S185.

ZHANG Y \& ZHANG G. 2009. One-step extrapolation method for reverse time migration. Geophysics, 74: A29-A33. 